\title{
DRONE CONTROL USING THE COUPLING OF THE PID CONTROLLER AND GENETIC ALGORITHM
}

Mohamed Elajrami*, Zouaoui Satla, Kouider Bendine

Laboratory of Mechanical Structures and Solids, University Djillali Liabes of Sidi Bel Abbes, Sidi Bel Abbes, Algeria

*E-mail of corresponding author: eladjrami_mohamed@yahoo.fr

\section{Resume}

Due to their strong abilities and easy usage, unmanned aerial vehicles (UAVs) commonly named drones have found a place and merged in varieties of industrial sectors. These diversities of applications have encouraged researchers to involve new control algorithms that offer the drones to gain more maneuverability and flexibility. In this regard, the present study aims to propose a new PID controller with optimally selected gain values. The control algorithm has been used for the case of drones' type quadcopter. To this purpose, a state-space representation have been formulated based on the Newton Euler's rigid body method. As an enhancement of the performance of the control algorithm (PID) the parameters $\mathrm{Kp}, \mathrm{Ki}$ and $\mathrm{Kd}$ have been selected thanks to an optimization search scheme based on a genetic algorithm. Various simulations were performed to test validity of the proposed model.

Available online: https://doi.org/10.26552/com.C.2021.3.C75-C82

\section{Article info}

Received 18 July 2020

Accepted 2 December 2020

Online 20 April 2021

\section{Keywords:}

drone,

control,

PID,

genetic algorithm

ISSN 1335-4205 (print version)

ISSN 2585-7878 (online version)

\section{Introduction}

Unmanned air vehicles, shortly called UAVs, have found application in unlimited sectors ranging from military to civil world. They can serve in different areas, which include, but not limited to, capturing photos, monitoring pipelines and megastructures, inspection missions, neutral military targets. The UAVs nowadays are almost everywhere which results in an increasing interest in their designing, modeling and monitoring that led to a great enhancement in their performance. The UAVs range in shape, size, power-driven and control system, among this diversity, one comes across the well know Quadcopters. The Quadcopters by definition refer to a multi rotor helicopter that uses four rotors with propellers to provide lifting. Disregarding the fact that Quadcopters are not as fast as other types of UAVs, however, they provide a great advantage, which is mainly the Vertical Take-Off and Landing concept that turns to be critical in a specific type of missions and operations. Recently, a focus has been made on the Quadcopters, this interest has been motivated by the aforementioned advantages. Thus, a lot of papers have been dedicated to the modeling of Quadcopters. Some of the literature sources have offered various models for Quadcopters, which are diverse by their complexity and included parameters [1-6].

The main two approaches used to formulate the mathematical representative model are the Newton-
Euler and Euler-Lagrange. Both approaches result in different types of models that range from the statespace representation to a transfer functions, which are later coupled with different control algorithms to reach the required maneuverability. The research community have investigated these approaches widely for more details about the formulations reader can refer to [7]. The authors proposed a mathematical model that relies on the Euler Newton formula and a simple PID controller for the case of quadcopter. The model steering angles were changed based on a fixed frame, which helps simplifying the final dynamic model, thus leading to an easy control of the drone [8]. Szabolcsi [9] introduces an analytic method with an enhanced PID and PI controllers for the case of the drone autopilot; the author's work was mainly based on MATLAB scripting. A method to fairly predict heading pitch and roll angle, using polynomial and logarithmic method, is presented in [10].

Obviously, the control algorithms are crucial for the UAV models development, they are considered as the brain that serves to control the structures and deliver the required path. Various techniques of control were tested for examined behavior of the UAV quadrotors, ranging from classical like PID to optimal like LQR, LQG [7] or fuzzy [11]. Each proposed controller has its advantage and a draw back. Optimal controllers, such as LQR and LQG, have a great performance and less noises. However, unfortunately, to be 


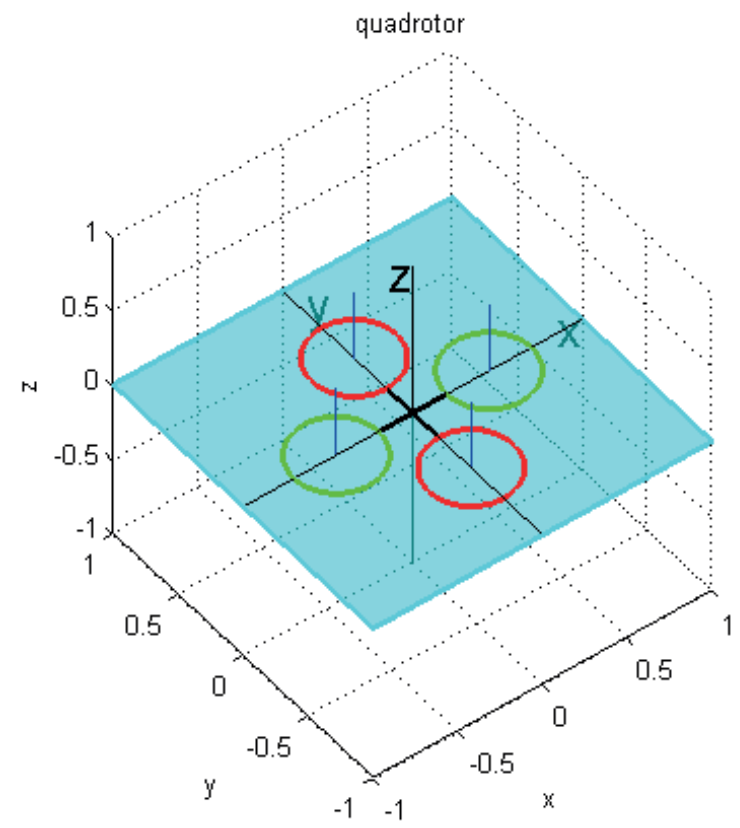

Figure 1 Quadcopter and principal axes

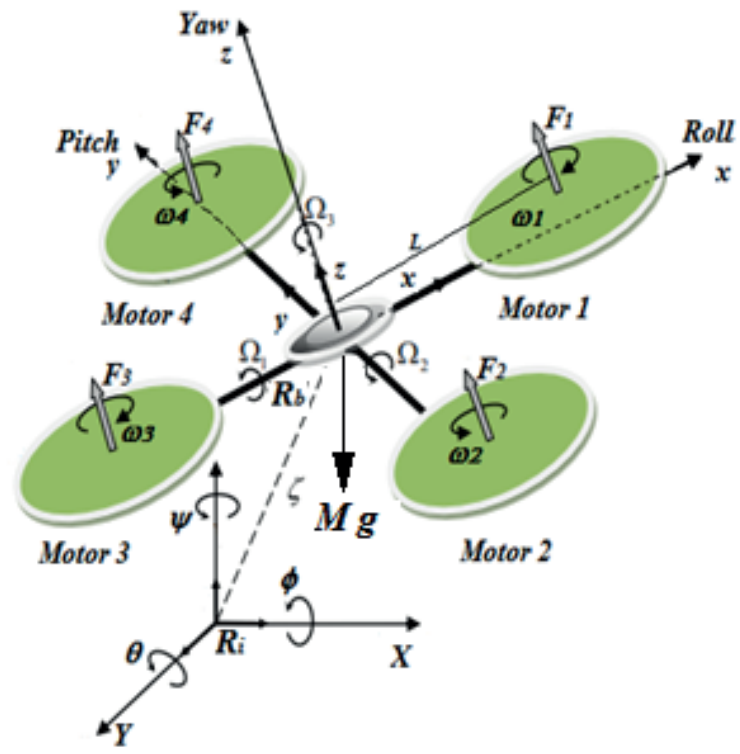

Figure 2 The structure of quadrotor and relative coordinate systems

used the model needed to be properly defined and mathematically formulated, which is not easy, especially when the models were of the complex type and also needed a parameters tunning. On the other hand, controllers like PID are extremely easy to implement and required no knowledge of the model. Sadly, the PID is less suitable for the nonlinear models and provide less performance. Generally, the choice of the controller is mainly driven by the used model and an enhancement is always needed to reach the desired performance and this paper is a contribution to that enhancement.

In terms of usability, both methods are fully accurate in simulation analysis but limited when it comes to the real-life models and the need for parameters' identification is crucial.

In this paper, the Newton Euler method has been used to formulate the state-space equation; the obtained equation involved six coordinates, which is then reduced to a more soft model that includes three main coordinates, which are the altitude $z$, steering angle (pitch), the direction of an arrangement parameter ( $Y$ coordinates). The state space equation is then coupled with a PID controller to easy monitor the drone. To improve the efficiency, the controller parameters were chosen based on a searching optimization scheme with help of the genetic algorithm (GA) in a way that has not been studied before. The angle has a range between 
values 0 and 10 degrees and this leads to a significant simplification of the equation, as one can notice in the next section. The optimization objective function has been taken to be the minimum of the difference between the desired position and the actual one. Different scenarios have been discussed and a conclusion is formulated.

\section{Mathematical formulation}

A good mathematical model representation can help improving the controllability of the drone. One of the best formulation methods that proves to be efficient is the well-known Newton-Euler. Thus, the proposed model is based on this formulation and the following dynamic equation is written [8]:

$$
\left[\begin{array}{cc}
M I_{3 \times 3} & 0 \\
0 & I
\end{array}\right]\left[\begin{array}{c}
\dot{V} \\
\dot{\Omega}
\end{array}\right]+\left[\begin{array}{c}
\Omega \times M \dot{\xi} \\
\Omega \times I \dot{\eta}
\end{array}\right]=\left[\begin{array}{c}
F \\
\tau
\end{array}\right]
$$

The model (Figure 1) is represented by 12 state variables, describing the quadrotors dynamic behavior: the position $\xi=[X Y Z]$, the linear velocity $V=[u v w]$ and, rotational angles $\eta=[\phi \theta \psi]$ (Roll, Pitch and Yaw) and angular velocities $\Omega=\left[\Omega_{1} \Omega_{2} \Omega_{3}\right]$. The vector $(F, \tau)$ stands for the applied forces and torques generated by the four motors rotation, while $M$ is the quadcopter mass and $M$ is the inertia.

Including the effect of the gravitational force and the vector of controller inputs are shown in Figure 2.

The dynamic model Equation (1) of a drone is driven using the Newton-Euler approach; a simplified mathematical model has been chosen [12]. The differential equation of motion of the modelled can be formulated as follows:

$$
\left\{\begin{array}{c}
\ddot{X}=(\sin \phi \sin \phi+\cos \phi \sin \theta \cos \phi) \frac{U_{1}}{M} \\
\ddot{Y}=(\sin \phi \cos \phi+\sin \phi \sin \theta \cos \phi) \frac{U_{1}}{M} \\
\ddot{Z}=-g+(\cos \phi \cos \theta) \frac{U_{1}}{M} \\
\ddot{\phi}=\frac{U_{2}}{I_{x x}} \\
\ddot{\theta}=\frac{U_{3}}{I_{y y}} \\
\ddot{\phi}=\frac{U_{4}}{I_{z z}}
\end{array} .\right.
$$

\section{The control law}

The PID algorithm is considered as the simplest controller, not just for its easy implementation, but for the high efficiency and reliability, as well. The PID controller can be formulated as follows [4]:

$$
u(t)=K_{p} e(t)+K_{i} \int e(t) d t+K_{d} \dot{e}(t),
$$

where:

$K_{p}:$ is the proportional gain,

$K_{i}:$ is the integral gain,

$K_{d}:$ is the derivation gain.

$e(t)$ : is the error given in the case of the drone by:

$e(t)=s_{p}-p_{v}(t)$

where:

$s_{p}:$ is the setpoint or desired position,

$p_{v}(t)$ : is the process variable at an instantaneous time according to $s_{p}$

\section{Results and discussion}

To study the efficiency of the proposed methodology, a simulation study has been carried out using Matlab Simulink for 3 degrees of freedom. The model is presented in Equation (5). The physical parameters of the proposed quadcopter can be found in [13-14]. Three PID controllers are implemented to control the pitch, the altitude $z$ and the y coordinates. To optimally choose parameters $K_{p}, K_{i}$ and $K_{d}$ a GA (genetic algorithm) optimization technique has been used to minimize the error between the desired drone position and the real one. After tuning the optimization algorithm, the following PID parameters were obtained:

$\left\{\begin{array}{c}\ddot{Y}=(\sin \phi) \frac{U_{1}}{M} \\ \ddot{Z}=-g+(\cos \phi) \frac{U_{1}}{M} . \\ \ddot{\phi}=\frac{U_{2}}{I_{x x}}\end{array}\right.$

After considering the pitch angle to take the values between 0 and $10(0<\emptyset<10)$, it is possible to linearize equation (5), by substituting $\sin \emptyset$ with $\emptyset$ and $\cos \emptyset$ with 1, which leads to Equation (6). Then the model can be easily designed as a block scheme using the Matlab Simulink, as shown in Figure 3.

$$
\left\{\begin{array}{c}
\ddot{Y}=(\phi) \frac{U_{1}}{M} \\
\ddot{Z}=-g+\frac{U_{1}}{M} . \\
\ddot{\phi}=\frac{U_{2}}{I_{x x}}
\end{array}\right.
$$

\subsection{Optimization with genetic algorithm (GA)}

To extract the needed PID parameters for the case of pitch angle controlling, each parameter was isolated separately to accelerate the solution calculation and the first parameter that has been optimized is the proportional gain $K_{p}$. As presented in Figure 4, the optimization results a value of proportional gain $K_{p}$ equal 


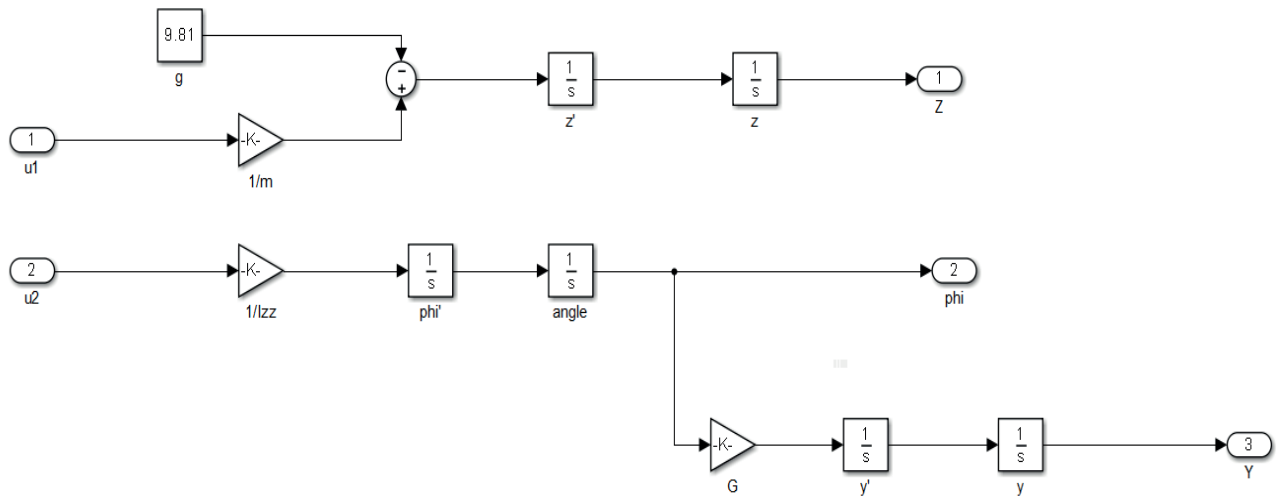

Figure 3 The block diagram of a quadrotor with $3 D D L$
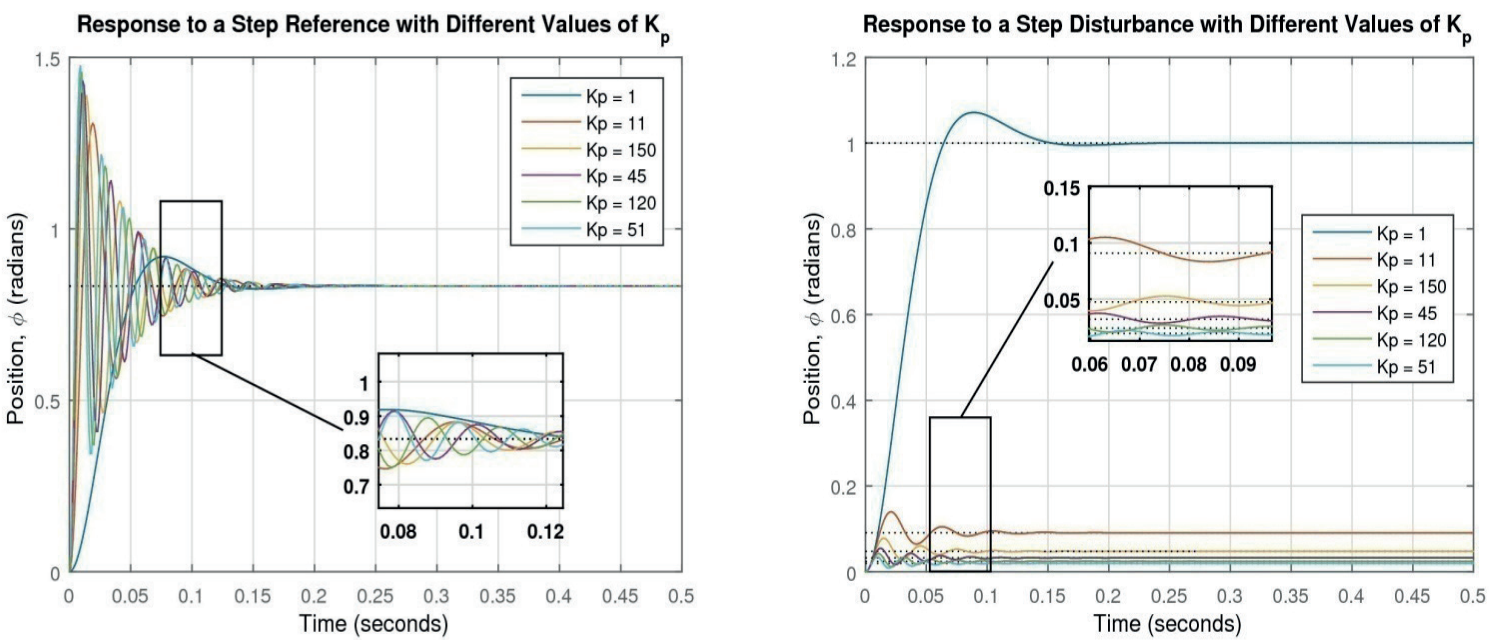

Figure 4 Optimization of the $K_{p}$ parameter of the PID controller of a pitch angle, $K_{p}=150$
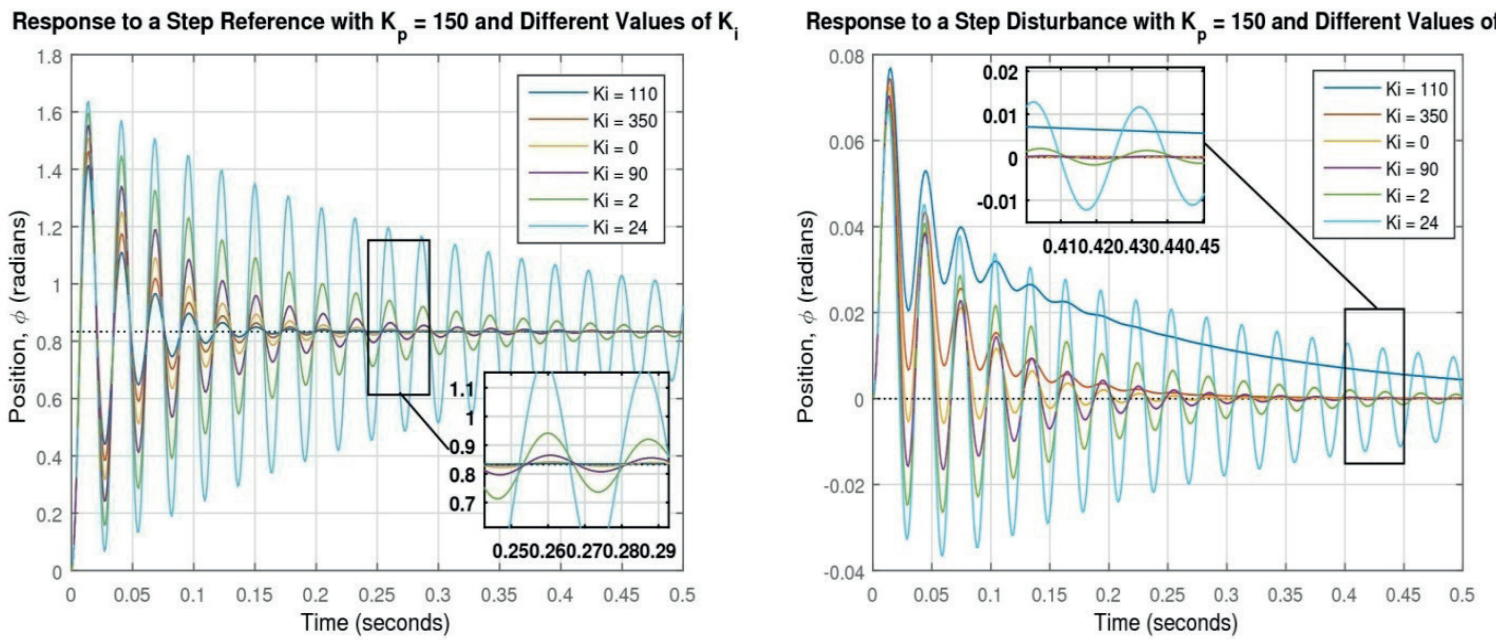

Figure 5 Optimization of the $K_{i}$ parameter of the PID controller of a pitch angle, $K_{i}=0$

to 150 . After, the first optimization the model shows some disturbance. Hence, an integral parameter has been plugged to reduce the resulting disturbance. The second optimization is then set, maintaining the $K_{p}$ as 150 and optimizing the $K_{i}$ gain for the set interval of [0,350].
The integral control has successfully reduced the steady-state error to zero and test integral gains $K_{i}$ ranging from 0 to 350 , even when a step disturbance is present (Figure 5); that was the aim for accumulation of the integral term. For the reaction to 

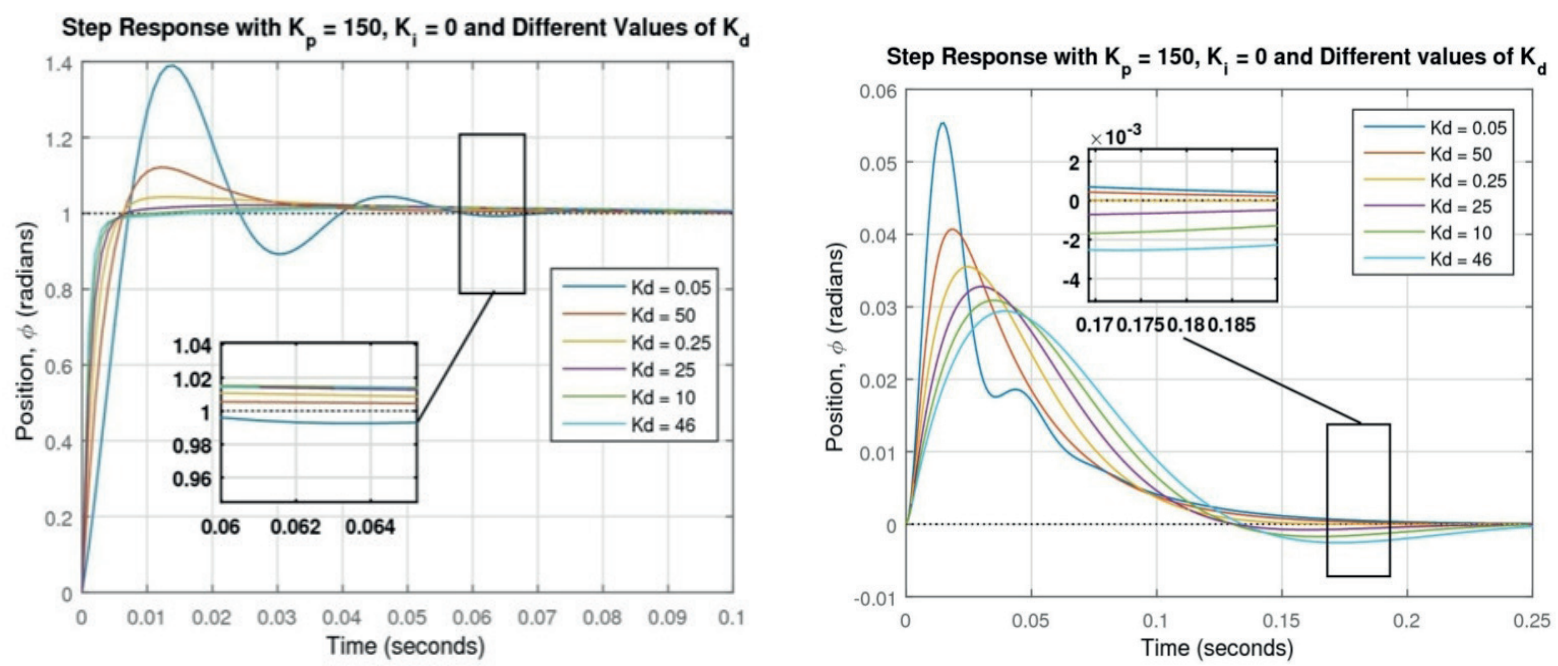

Figure 6 Optimization of the $K_{d}$ parameter of the PID controller of a pitch angle, $K_{d}=50$

Table 1 Optimization of parameters of the PID controller in the $z$ and $y$ directions

\begin{tabular}{cccc}
\hline parameters PID controller & $K p$ & $K i$ & $K d$ \\
\hline PID in the z-direction & 5 & 4 & 3 \\
PID in the y-direction & 0.3 & 0 & 10 \\
\hline
\end{tabular}

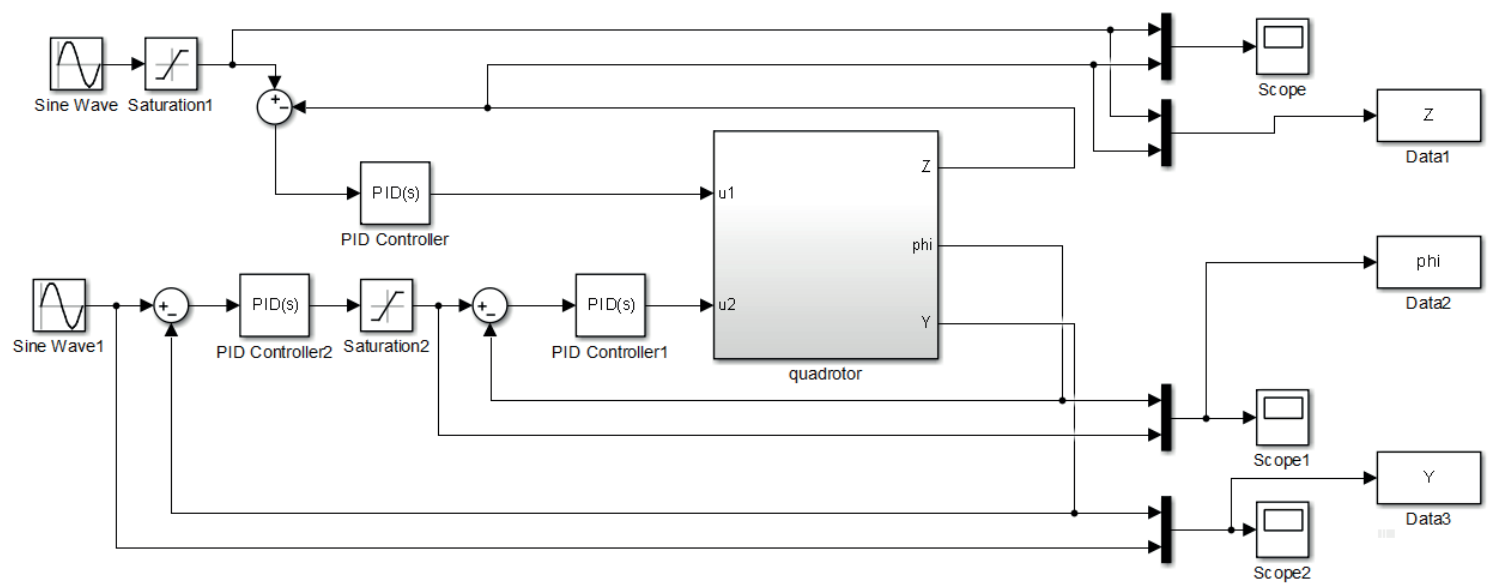

Figure 7 The block diagram of the closed-loop system

the step reference, all of the responses look similar to the amount of oscillation, increasing slightly as $K_{i}$ is made it better, though, the response due to the disturbance changes considerably as the integral gain $K_{i}$ is changed. Exclusively, the larger the value of $K_{i}$ employed, the faster the error decays to zero. The best choice for a value of $K_{i}$ will be $K_{i}=0$ because the error due to the disturbance decays to zero quickly, however, the response to the reference has a longer settling time and more overshoot.

Introducing the term derivative to the control will help reducing the time needed for the stability and overshoot. Adding a derivative term to the console is the final step to reach the full working PID controller. The derivative gains that range from 0.05 to 50 were investigated by fixing the two proportional and integral terms with their previously selected values of an optimization $K_{p}=150$ and $K_{i}=0$.

Figure 6 also shows that when $K_{d}=50$, the full requirements for the pitch angle control have been met.

In the same way, the values of the terms proportional to the integral and derivative of the two directions $z$ and $y$ are extracted, as shown in Table 1 . There is an explanatory point since angle $\emptyset$ has a direct effect on $y$. Must first know The behavior of angle must be first known; thus it became known in advance in the field of simulation, which made it easy to study the behavior of $y$.

After including all the physical and the controller parameters and by using the aforementioned equations a Simulink block has been designed, as shown in Figure 7 . 
The proposed controller is tested with the following desired paths of the drone:

$Z_{\text {desired }}=\sin (t)$,

$Y$ desired $=\sin \left(\frac{1}{2} * t\right)$.

The model is run for time of $25 \mathrm{~s}$. The obtained results are shown in Figures 8 to 10. Figure 8 presents the pitch angle variation over time. From the figure can be seen that there is a small error in the start of the simulation and that is because the controller is trying to catch the desired position. Once the actual position coincided with the desired one, the curve is stabilized, which is a proof of the proposed controller accuracy. The same observation is suitable for the $y$ and $z$ coordinate in Figures 8 and 9. For the better understanding of the proposed path, a 3D curve is presented in Figure 11. The figure reveals without doubts that the drone is following the same trajectory as the predicted one.

As a study of the performed optimization, the time needed to extract each PID parameter has been calculated and presented in table 2 . It is worthy to note here that the used computer is with performance of the CPU i5 and 4G Ram. As it is clearly shown in Table 2, the required time for the whole optimization is indeed negligible.

\section{Conclusion}

The present study aims to provide a mathematical model with a simple control scheme for the case of a small quadrotor. The model is formulated using the Newton-Euler assumption and extracted

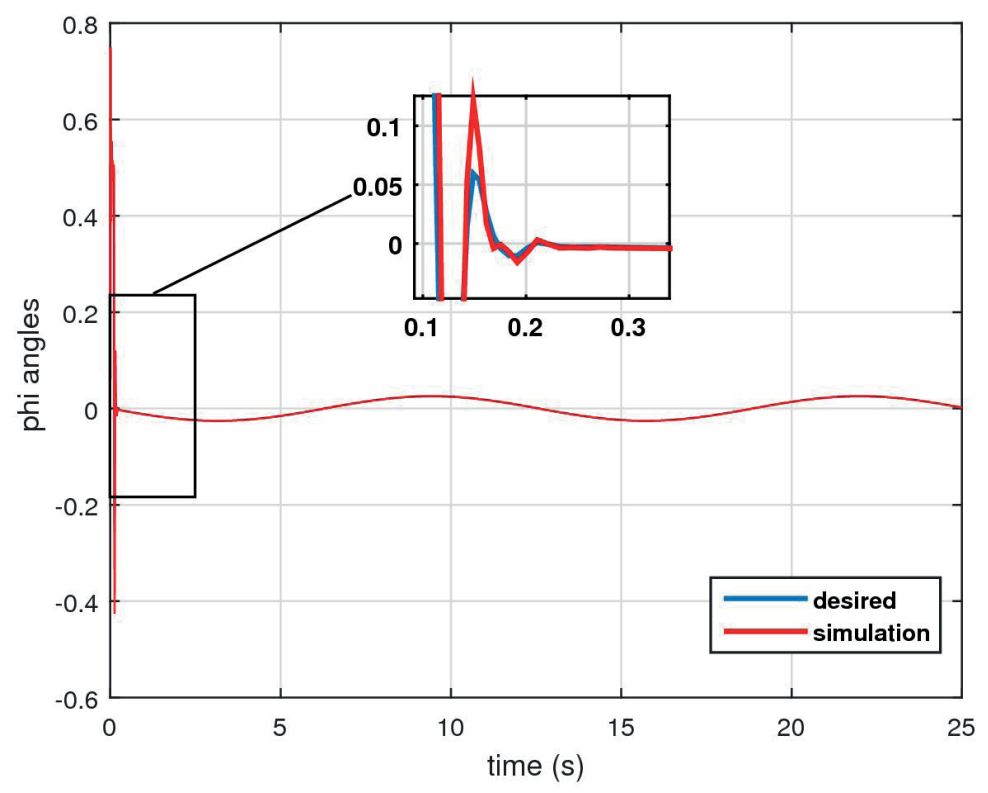

Figure 8 The desired trajectory of the pitch angle and actual response

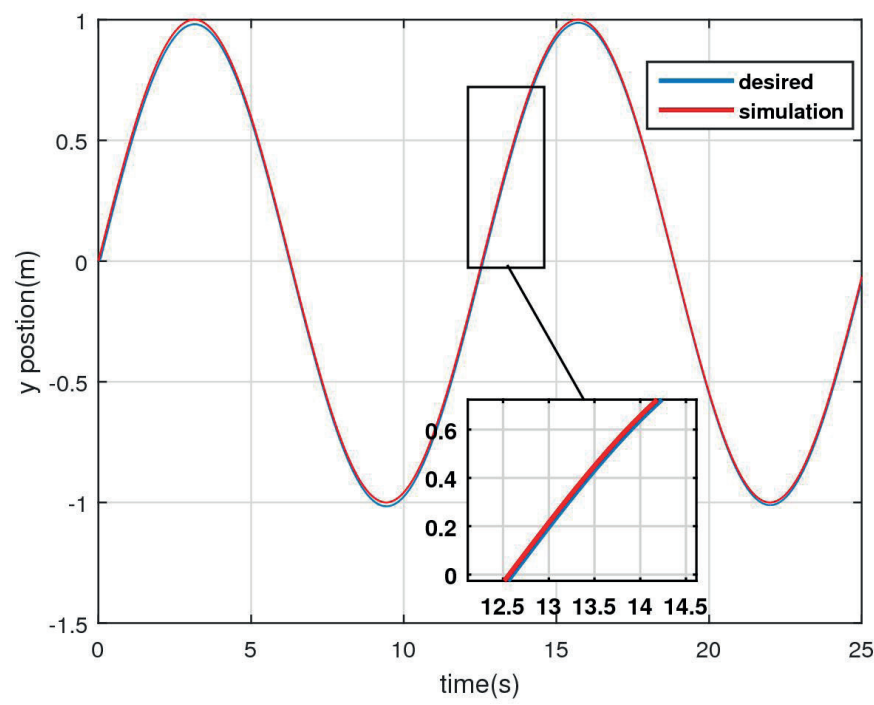

Figure 9 The Y position response of the closed-loop system 


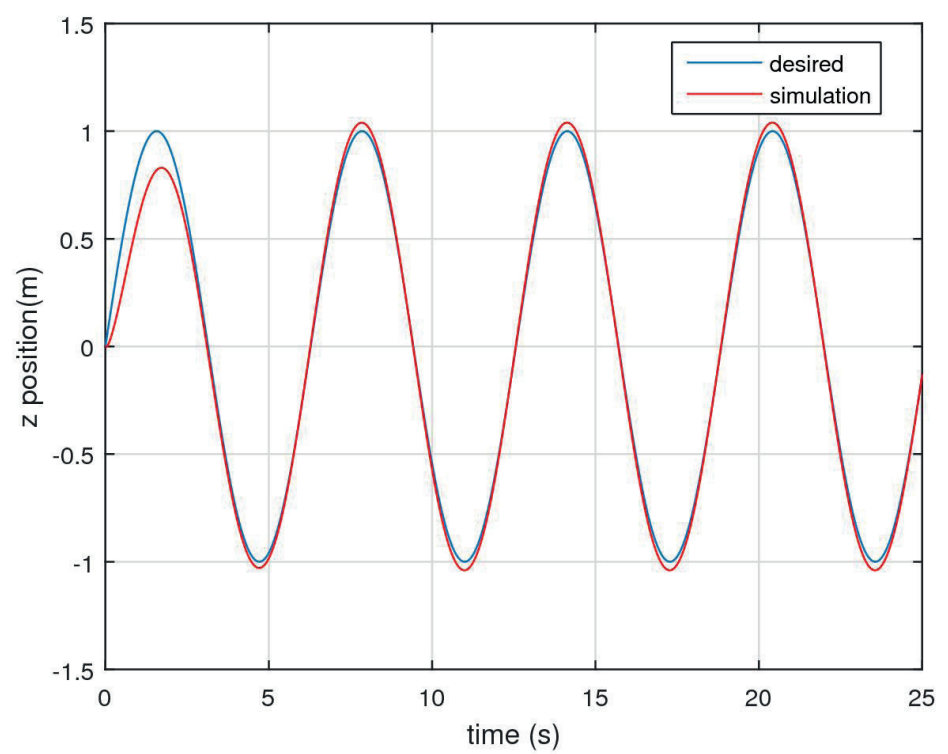

Figure 10 Response of the closed-loop system to the desired trajectory of altitude

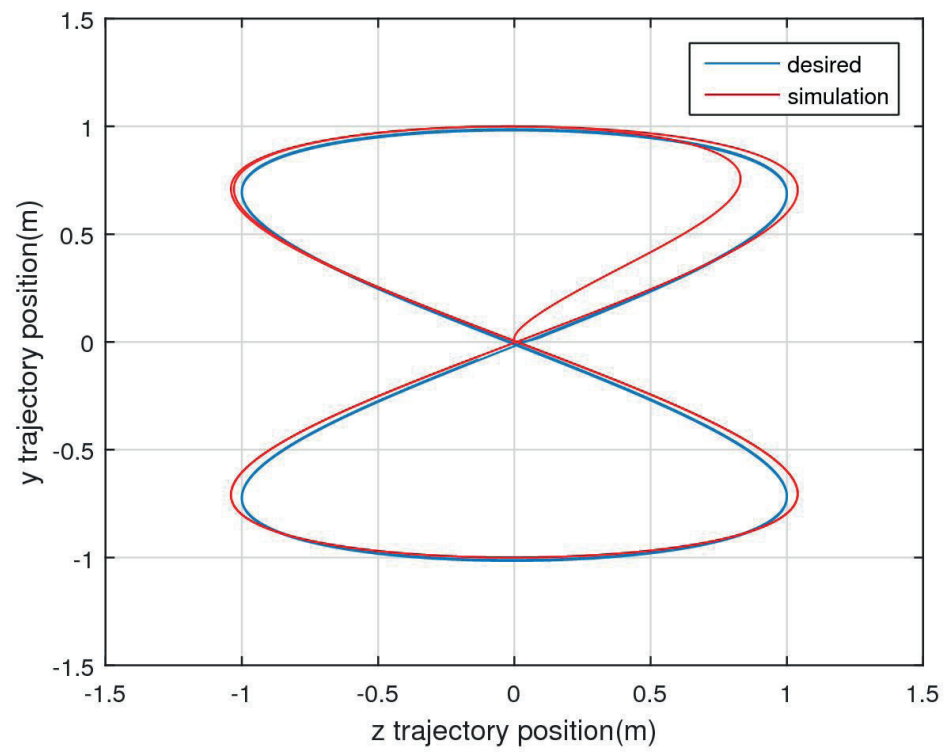

Figure 11 The closed-loop system response with the PID trajectory $3 D$ controllers

Table 2 The CPU time needed for the optimization routine

\begin{tabular}{cccc}
\hline parameters controller & $\emptyset(\mathrm{s})$ & $z(\mathrm{~s})$ & $y$ \\
\hline CPU time for optimization of parameters PID & 7731.605431 & 6605.553147 & 7533.582554 \\
CPU time for simulation & & 1815.246054 & \\
\hline
\end{tabular}

based on a dynamic equation. To control the drone a PID algorithm has been designed with considering use of the best tuning parameters. The controller parameters have been selected using the Genetic Algorithm (GA) search optimization scheme.

To test the accuracy of the proposed model, a simulation study has been carried out for $25 \mathrm{~s}$. The error between the desired and simulation trajectory is reduced successfully to a very low value for the three control parameters, as well as the altitude, which proves the robustness towards the stability and tracking of the proposed model.

The results of this paper show that this method of controlling the quadrotor has a very high performance to track the position path and hover and a good stability of this model. 


\section{References}

[1] AL YOUNIS, Y., AL JARRAH, M. A., JHEMI, A. A. Linear vs. nonlinear control techniques for quadrotor vehicle. In: 7th Symposium of Mechatronics and its Applications ISMA 2010: proceedings. IEEE. 2010.

[2] MIAN, A. A., DAOBO, W. Nonlinear flight control strategy for an under actuated quadrotor aerial robot. In: $15^{\text {th }}$ IEEE International Conference on Networking, Sensing and Control ICNSC: proceedings [online]. IEEE. 2008. ISBN 978-1-4244-1685-1. Available from: https://doi.org/10.1109/ICNSC.2008.4525351

[3] MAHONY, R., KUMAR, V., CORKE, P. Multirotor aerial vehicles modeling simulation and control of quadrotor. IEEE Robotics and Automation Magazine [online]. 2012, 19(3), p. 20-32. ISSN 1070-9932. Available from: https:// doi.org/10.1109/MRA.2012.2206474

[4] KOSZEWNIK, A. The parrot UAV controlled by PID controllers. Acta Mechanica et Automatica [online]. 2014, 8(2), p. 65-69. eISSN2300-5319. Available from: https://doi.org/10.2478/ama-2014-0011

[5] JAMKHANDI, A. G., TULPULE, S., CHATURVEDI, A., CHARVET, J.-N. Controlling the position and velocity in space of the quad-rotor UAV AR. Drone using predictive functional control and image processing in open CV. In: 2012 International Conference on Signal Processing Systems ICSPS 2012: proceedings [online]. IPCSIT. Vol. 58. Singapore: IACSIT Press, 2012. Available from: https://doi.org/10.7763/IPCSIT.2012.V58.3

[6] SALIH, A. L., MOGHAVVEMI, M., MOHAMED, H. A. F., GAEID, K. S. Flight PID controller design for a UAV quadrotor. Scientific Research and Essays. 2010, 5(23), p. 3660-3667. ISSN 1992-2248.

[7] ALTUG, E. Vision-based control of unmanned aerial vehicles with applications to an autonomous four-rotor a helicopter, quadrotor. Ph.D. thesis, University of Pennsylvania, 2003.

[8] SATLA, Z., ELAJRAMI, M., BENDINE, K., SALAH, M., POLAT, A. P, PI, PID controller designed for UAV Quadrotors trajectory. In: International Conference on Innovative Engineering Applications: proceedings. 2018.

[9] SZABOLCSI, R. Optimal PID controller-based autopilot design and system modelling for small unmanned aerial vehicle. Review of the Air Force Academy [online]. 2018, 3(38), p. 43-58. ISSN 1842-9238. Available from: https://doi.org/10.19062/1842-9238.2018.16.3.6

[10] WIERZBICKI, D., KRASUSKI, K. Methods of predicting the heading, pitch and roll angles for an unmanned aerial vehicle. Communications - Scientific Letters of the University of Zilina [online]. 2020, 22(2), p. 52-59. ISSN 1335-4205, eISSN2585-7878. Available from: https://doi.org/10.26552/com.C.2020.2.52-59

[11] AMIRKHANI, A., SHIRZADEH, M., KUMBASAR, T. Interval type-2 fuzzy cognitive map-based flight control system for quadcopters. International Journal of Fuzzy Systems[online]. 2020, 22, p. 2504-2520. ISSN 15622479, eISSN 2199-3211. Available from: https://doi.org/10.1007/s40815-020-00940-8

[12] KHATOON, S., SHAHID, M., CHAUDHARY, H. Dynamic modeling and stabilization of quadrotor using PID controller. In: International Conference on Advances in Computing, Communications and Informatics ICACCI 2014: proceedings [online]. IEEE. 2014. p. 746-750. Available from: https://doi.org/10.1109/ICACCI.2014.6968383

[13] MOHAMMED, M. J., RASHID, M. T., ALI, A. A. Design optimal PID controller for quad rotor system. International Journal of Computer Applications [online].2014, 106(3), p. 15-20. ISSN 0975-8887. Available from: https://doi.org/10.5120/18500-9565

[14] SATLA, Z., ELAJRAMI, M., BENDINE, K. Easy tracking of UAV using PID Controller. Periodica Polytechnica Transportation Engineering [online]. 2019, 47(3), p. 171-177. ISSN 0303-7800, eISSN1587-3811. Available from: https://doi.org/10.3311/PPtr.10838 